\title{
Body Mass Index and Risk for Mental Stress-Induced Ischemia in Coronary Artery Disease
}

\author{
Robert Soufer, ${ }^{1,2^{*}}$ Antonio B Fernandez, ${ }^{1^{*}}$ Judith Meadows, ${ }^{1,2}$ Dorothea Collins, ${ }^{2}$ and Matthew M Burg ${ }^{1,2}$ \\ ${ }^{1}$ Section of Cardiovascular Medicine, Yale School of Medicine, New Haven, Connecticut, United States of America; and \\ ${ }^{2}$ VA Connecticut Healthcare System, West Haven Campus, West Haven, Connecticut, United States of America
}

\begin{abstract}
Acute emotionally reactive mental stress (MS) can provoke prognostically relevant deficits in cardiac function and myocardial perfusion, and chronic inflammation increases risk for this ischemic phenomenon. We have described parasympathetic withdrawal and generation of inflammatory factors in MS. Adiposity is also associated with elevated markers of chronic inflammation. High body mass index (BMI) is frequently used as a surrogate for assessment of excess adiposity and associated with traditional coronary artery disease (CAD) risk factors and CAD mortality. BMI is also associated with autonomic dysregulation and adipose tissue-derived proinflammatory cytokines, which are also attendant to emotion-provoked myocardial ischemia. Thus, we sought to determine if $\mathrm{BMI}$ contributes to risk of developing myocardial ischemia provoked by MS. We performed a prospective interventional study in a cohort of 161 patients with stable CAD. They completed an assessment of myocardial blood flow with single-photon emission computed tomography simultaneously during two conditions: laboratory MS and at rest. Multivariate logistic regression determined the independent contribution of BMI to the occurrence of MS-induced ischemia. Mean age was $65.6 \pm 9.0$ years, $87.0 \%$ had a history of hypertension and $28.6 \%$ had diabetes. Mean BMI was $30.4 \pm 4.7$. Prevalence of MS ischemia was $39.8 \%$. BMI was an independent predictor of MS ischemia, odds ratio $(\mathrm{OR})=1.10,95 \%$ confidence interval $(\mathrm{Cl})(1.01-1.18)$ for 1-point increase in $\mathrm{BMI}$ and $\mathrm{OR}=1.53,95 \% \mathrm{Cl}(1.06-2.21)$ for a 4.7-point increase in BMl (one standard deviation beyond the cohort BMI mean), $p=0.025$ for all. These data suggest that BMI may serve as an independent risk marker for MS ischemia. The factors attendant with greater BMl, which include autonomic dysregulation and inflammation, may represent pathways by which high BMI contributes to this risk and serves as a conceptual construct to replicate these findings in larger CAD populations.
\end{abstract}

Online address: http://www.molmed.org

doi: $10.2119 /$ molmed.2016.00128

\section{INTRODUCTION}

Mental and emotional stress can trigger acute coronary syndrome (ACS), but more often it provokes asymptomatic myocardial ischemia (1-3), a syndrome associated with an independent, 2.4- to three-fold increased risk of early morbidity and mortality in patients with known coronary artery disease (CAD) (4-7). The pervasive nature of this syndrome highlights its clinical importance, yet the fact that it is largely asymptomatic makes the identification of the vulnerable patient particularly challenging. Thus, the determination of risk markers for mental stress (MS)-provoked myocardial ischemia has significant implications for cardiovascular disease risk management and improved outcomes.

MS-provoked ischemia is distinguished by several unique pathophysiologic features. In particular, it is

*RS and ABF should be considered as co-first authors.

Address correspondence to Robert Soufer, Section of Cardiovascular Medicine, Yale University School of Medicine/VA Connecticut Healthcare System, 950 Campbell Avenue 111B, West Haven, Connecticut 06516, USA. Phone: 203-932-5711 ext. 3268; Fax: 203-937-3884; E-mail: Robert.soufer@yale.edu. Submitted May 10, 2016; Accepted for publication May 12, 2016; Published Online (www.molmed.org) May 19, 2016.

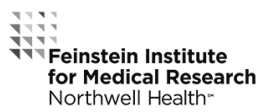

Feinstein Institute
for Medical Research Northwell Health"

characterized by a transient increase in coronary vasomotion that creates a dynamic coronary obstruction $(8,9)$ and is associated with a distinct autonomic pattern that has also been found by others to acutely increase circulating inflammatory markers derived from macrophages termed the parasympathetic inflammatory reflex (10).

High body mass index (BMI) is a frequently used surrogate for assessment of excess adiposity and associated with both traditional CAD risk factors and all-cause and CAD mortality. BMI is also associated with autonomic dysregulation (11), adipose tissue-derived proinflammatory cytokines and vasoactive peptides that have actions in the central nervous system and peripheral organs (12). Even with a modest increase in $\mathrm{BMI}$, there is an increase in adipokines paralleled by histological and biochemical changes characteristic of inflammation (12) and by endothelial dysfunction, 
one of the earliest signs of clinical atherosclerosis (13). Obesity has also been associated with psychosocial predictors of cardiovascular prognosis, including depression, anxiety and hostility (14-16). Thus, the link between obesity/adiposity and CAD-related prognosis includes both physiological and psychosocial pathways.

The risk for developing myocardial ischemia during MS has been linked to many of the same biological and psychological variables described above with an increased BMI (8,17-19). We therefore sought to determine if BMI contributes to risk for developing myocardial ischemia during MS among patients with stable CAD.

\section{MATERIALS AND METHODS}

\section{Participants}

Patients with chronic stable CAD ( $n=161)$, documented by remote history of ACS, surgical or percutaneous revascularization and/or positive exercise myocardial perfusion study were recruited from the Cardiology outpatient clinics at Yale University Medical Center and VA Connecticut Healthcare System from February 2004 through October 2010. Patients with a diagnosis of myocardial infarction or unstable angina within 3 months of the study, surgical or percutaneous revascularization within 6 months of the study, major cardiac arrhythmia, decompensated congestive heart failure, incapacitating or life-threatening illness, major psychiatric disorder, substance abuse disorder (by history) or cognitive impairment, pregnancy and/ or inability to speak or read English were excluded. The Institutional Review Board at both medical facilities approved the study, and all participants provided informed consent.

Medical chart review and patient interview were used to obtain demographic information and determine cardiovascular risk profile. Participants with a history of systolic pressure $>140$ $\mathrm{mmHg}$ or diastolic pressure $>90 \mathrm{mmHg}$, or currently taking medication for high blood pressure, were classified as hypertensive. Use of $\beta$-blockers, statins, angiotensin-converting enzyme inhibitors (ACE inhibitor), aspirin, calcium channel blockers and tobacco use was documented.

\section{Procedures}

Patients reported to the Cardiovascular Neurobehavioral Medicine Research Laboratory at 9 a.m. on the day of study. Participants were asked to eat a light breakfast and take their normal medications, and adherence to this was confirmed on their arrival. Height and weight were assessed, after which participants were instrumented with 12-lead electrocardiogram (EKG) monitor (GE Medical MAC 5500) and an automated blood pressure cuff. An indwelling intravenous catheter was placed for nuclear isotope injections and for the collection of blood samples. Myocardial perfusion was assessed at rest and during MS by single-photon emission computed tomography (SPECT) with Technetium-99 (Tc-99) tetrofosmin using a 1-d rest-stress protocol. The resting perfusion scan was performed 45 min after an intravenous injection of 10-12 mCi Tc-99m tetrofosmin (Myoview; GE Healthcare). After completion of the resting imaging scan, the participants were placed in a quiet room with dimmed lights in a relaxed semi-supine position. Participants then underwent the standardized laboratory MS protocol for our laboratory. The procedure has been previously described $(17,19)$. Briefly, this is comprised of a resting baseline (BL) during which patients are instructed to imagine being in a restful setting (10 $\mathrm{min})$, followed by a laboratory MS period (8 $\mathrm{min}$ ).

At the end of BL, a 4-mL blood sample was collected into refrigerated tubes containing reduced glutathione (for rest catecholamine assay) and placed on ice. The 8-min MS condition was then initiated. For this condition, mental arithmetic-serial subtraction-was used, as it is a particularly potent stress task for both male and female patients of diverse backgrounds $(19,20)$. Starting with a 4-digit number, patients were instructed to subtract a specified number (e.g., 7) serially. Following a standard protocol, the patient was instructed to work as quickly and accurately as they could, and they were both frequently prompted to work faster and firmly corrected when they made errors. The frequency of prompts was titrated to incur an error rate of 1 per 10 calculations, as we have previously described $(19,20)$, and this was achieved with all patients. Approximately $90 \mathrm{~s}$ into the MS condition, 30-32 mCi Tc-99m tetrofosmin was injected, followed by a second 4-mL blood sample (for stress catecholamine assay), with acquisition of SPECT imaging approximately 45-60 min later. This was followed by a second 4-mL blood sample (for stress catecholamine assay). Upon completion of the MS intervention, the catheter was removed and the patients were de-instrumented. Approximately $45 \mathrm{~min}$ after 30-32 mCi Tc-99m tetrofosmin was injected as previously mentioned during the MS condition, patients underwent this second myocardial perfusion scan for the stress condition.

Monitoring during the laboratory protocol was identical to that done for clinical stress testing. In brief, heart rate (HR), systolic/diastolic blood pressure (SBP/DBP) and 12-lead EKG were obtained at 5-min intervals during BL and at 1-min intervals during MS. Indication for early termination of MS likewise was identical to clinical stress testing: angina or symptom equivalent, ST segment depression of greater than $3 \mathrm{~mm}$, drop in SBP or any arrhythmia. As routinely reported in the literature on MS and indices of myocardial ischemia, none of these indications occurred during the protocol.

Blood samples for catecholamine analysis were centrifuged for $10 \mathrm{~min}$ at $4^{\circ} \mathrm{C}$ in an EC Centra-MP4R refrigerated highspeed tabletop centrifuge (International Equipment Co.). Serum aliquots were obtained for the different time points and stored in $2-\mathrm{mL}$ cryovials at $-30^{\circ} \mathrm{C}$ until analysis. 


\section{Measurements}

BMI calculations were performed based on height and weight obtained upon the patient's arrival for the experiment. At the Yale General Clinical Research Center, catecholamines were analyzed by high-performance liquid chromatography (ESA Inc.) using electrochemical detection (Coulochem II) after alumina extraction.

\section{SPECT Myocardial Perfusion Imaging}

Radiotracer dosing, image acquisition and image processing were performed according to guidelines of the American Society of Nuclear Cardiology (21) and followed a protocol identical to that used clinically in the low-dose/high-dose single-day protocol with rest imaging performed prior to stress imaging. Gated SPECT was performed with use of a stepand-shoot acquisition on a Philips Forte $\gamma$ camera system with Gd-153 line source attenuation correction or on a GE Discovery SPECT/CT system with computed tomography (CT)-based attenuation correction. Data were acquired over $180^{\circ}$, with 64 frames with a $64 \times 64$ matrix, 8 frame gating and $20 \%$ window centered on $140 \mathrm{keV}$ photo peak of Tc-99m.

Tomographic images were reconstructed using standard filtered back projection and Butterworth low-pass filtering, using JET stream platform (Philips Medical Systems). Short- and long-axis SPECT slices were generated, along with multilevel gated cines. Reconstructed images were interpreted using AUTOQUANT (Cedars-Sinai). Myocardial perfusion images were independently analyzed and interpreted by two experienced nuclear cardiologists (RS and JM), blinded to the name and risk profile of the patients and all other medical data, including depression status. The concordance rate was 95\%, with discordant studies addressed by another joint review where consensus was reached.

Perfusion and wall motion were assessed in a qualitative and a semiquantitative manner. Perfusion images were analyzed in both a grayscale and color display. Rest and stress images were visually compared for number and severity of perfusion defects using a 17-segment model. Each segment was scored from 0 to 4 , with 0 being "normal uptake" and 4 being "no uptake," yielding a total score. A reversible defect score (summed difference score [SDS]) was calculated as the difference between summed stress (SSS) and rest scores (SRS). Following published guidelines (21), a new impairment in myocardial perfusion during stress compared with the resting BL images in a segment was defined as a score $\geq 2$ (definitely abnormal); a worsening impairment was defined as an increase in score $\geq 1$ above a score of 2 or greater. If any of the 17 segments evidenced these scores, the patient was categorized as having new / worsening impairment in myocardial perfusion during MS challenge.

\section{Data Analysis}

BMI was treated as a continuous variable. Change from rest to MS for cardiovascular (HR and SBP/DBP) and neuroendocrine (epinephrine and norepinephrine) indices were assessed with t-tests or sign rank tests as appropriate. Serum catecholamine levels in our population did not have a normal distribution; therefore, logarithmic transformation of those values was warranted. A multivariate logistic regression model was used to examine whether BMI independently predicted MS-provoked myocardial ischemia after adjusting for variables, whose selection was based on previous research. These variables included traditional cardiovascular risk factors (age, left ventricular ejection fraction [LVEF]); hypertension and diabetes based on the association of endothelial dysfunction in patient populations with either condition; $\beta$-blocker use because of its protective role for myocardial ischemia and endothelial function; and logarithmic transformations of resting serum levels of epinephrine and norepinephrine based on their potential role as mediators in MS ischemia. All tests were two-sided, and analyses were performed using SAS version 9.1.

\section{RESULTS}

The overall study population had the following demographic and clinical correlates' (see Table 1): average age was 66 years $( \pm 9), 87 \%$ were male, $93 \%$ had hyperlipidemia, $87 \%$ were hypertensive, $29 \%$ were diabetic and $25 \%$ were tobacco users; 70\% were prescribed aspirin, $93 \%$ a statin, $78 \%$ a $\beta$-blocker, $21 \%$ plavix, $50 \%$ an ACE inhibitor and 29\% a calcium channel blocker; and the mean LVEF was 54\%. All subjects were stable class 1 or 2 New York Heart Association (NYHA) classification with regard to symptoms. Thus, there were no statistically significant differences in these variables between those with versus without worsening impairment in myocardial perfusion from BL to MS.

\section{Effects of MS}

Of the 161 study participants, 64 $(39.8 \%)$ had a new or worsening myocardial perfusion defect from BL to MS, or MS-provoked myocardial ischemia, with a group mean SDS = 4.84 (vs. 0.92 in patients without MS-provoked myocardial ischemia) (Table 2). Patients with versus without this ischemia were comparable with regard to clinical manifestations of CAD, with no BL differences in primary risk factors, medications, comorbidities, LVEF, severity of CAD and prior revascularization, or hemodynamics (see Table 1).

At BL, the mean SBP was $133( \pm 18)$, mean DBP was $75( \pm 10)$ and mean HR was $59( \pm 11)$, while the mean LVEF derived from SPECT myocardial perfusion imaging (MPI) was $56.0( \pm 10.8)$. With MS, this increased to an SBP/DBP of $160( \pm 21) / 88( \pm 11)$ and HR of $69( \pm 12)$, while the SPECT MPI-derived LVEF was 56.9 ( \pm 11.6$)$. The apparent lack of change in LVEF from BL to post stress is not surprising since the post stress SPECT images were acquired 45-60 min after the stress. While the perfusion images acquired at that time reflect perfusion at the time of injection-for example, at the time of the stress-the gated wall motion images on which LVEF is derived reflect wall motion at the time of acquisition-for example, 45-60 min after 
Table 1. Patient characteristics.

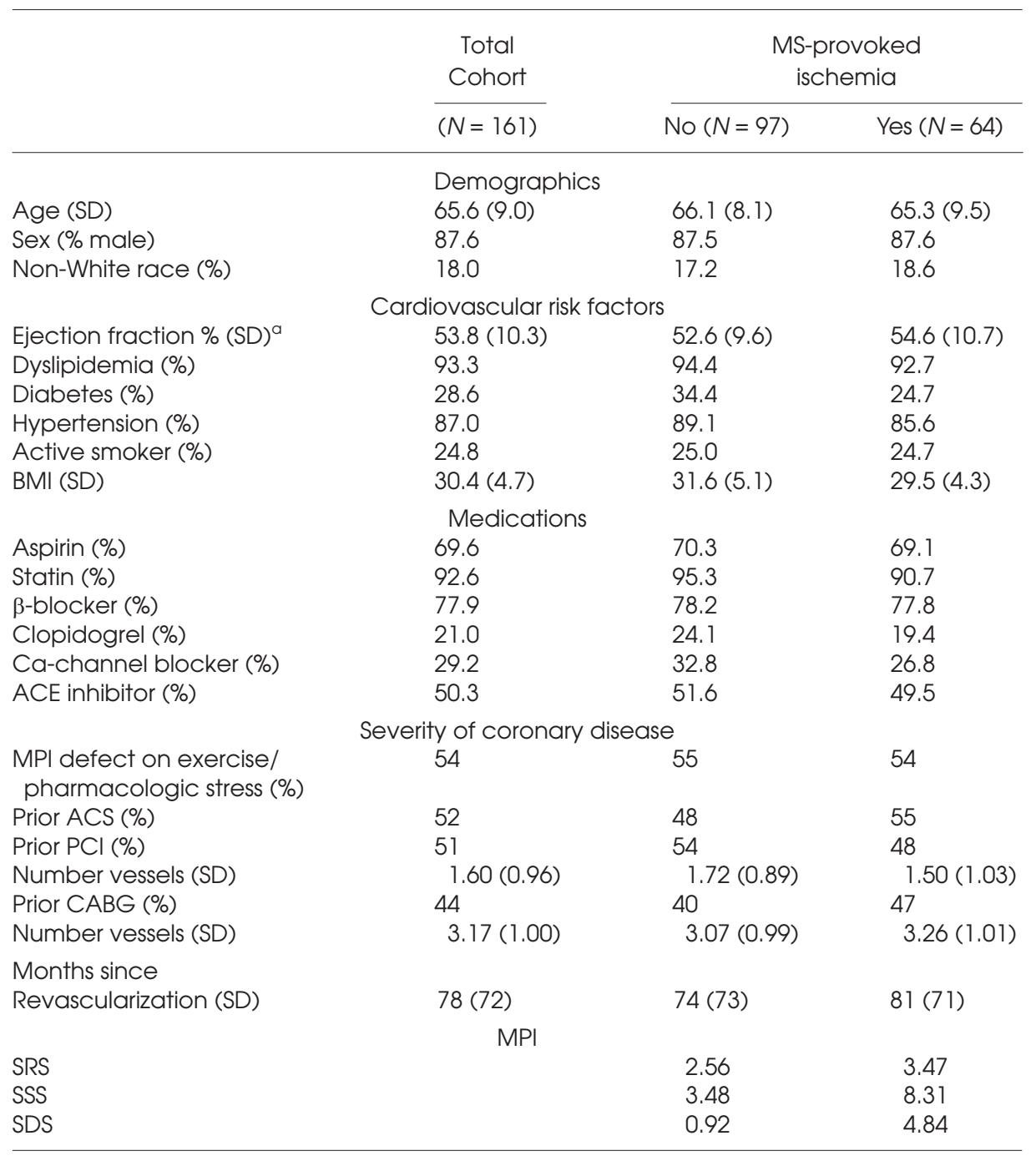

Ca, calcium; CABG, coronary artery bypass graft surgery; $\mathrm{PCl}$, percutaneous coronary intervention; SD, standard deviation.

aLVEF value taken from the most recent diagnostic test in the medical record (e.g., resting cardiac ECHO/SPECT MPI, cardiac catheterization). injection, a time when any deficits in LVEF provoked by psychological stress would be expected to have normalized. Patients with MS-provoked myocardial ischemia showed a trend of higher BL levels of circulating epinephrine $(35.0 \pm 50.6 \mathrm{pg} / \mathrm{mL}$ vs. $24.6 \pm 26.8 \mathrm{pg} / \mathrm{mL})$ and norepinephrine $(402.6 \pm 263.2 \mathrm{pg} / \mathrm{mL}$ vs. $341.0 \pm 254.9 \mathrm{pg} / \mathrm{mL}$ ) and a greater increase in these catecholamines with MS (epinephrine $74.6 \%$ vs. $51.6 \%$; norepinephrine $8.6 \%$ vs. $5.0 \%$ ) that did not reach significance (see Table 2 ).

Patients with MS-provoked myocardial ischemia had higher BMI (31.6 \pm 5.1 vs. $29.5 \pm 4.3$, respectively, $p=0.03$ ), and in the multivariate model, the likelihood of MS-provoked myocardial ischemia increased by $10 \%$ for each point increase in BMI (odds ratio [OR] $=1.10,95 \%$ confidence interval [CI] [1.01-1.18], $p=0.025)$, by $31 \%(\mathrm{OR}=1.31,95 \% \mathrm{CI}[1.04-1.66])$ for a 3-point increase in BMI and by 53\% $(\mathrm{OR}=1.53,95 \% \mathrm{CI}[1.06-2.21])$ for one standard deviation (4.7 points) beyond the BMI mean value in our cohort ( $p=0.025$ for all).

\section{DISCUSSION}

In this study, we found that BMI was associated with an increased likelihood for myocardial ischemia in response to a cognitive stressful trigger, which is MS ischemia. For each 1-point increase in BMI, individuals with stable CAD were found to have a $10 \%$ increased risk of evidencing this ischemic syndrome independent of traditional cardiovascular risk factors for CAD-related outcomes.

Table 2. Physiologic response to stress.

\begin{tabular}{|c|c|c|c|c|c|c|}
\hline \multirow[b]{3}{*}{ Measure } & \multirow{2}{*}{\multicolumn{2}{|c|}{$\begin{array}{l}\text { Overall } \\
(N=161)\end{array}$}} & \multicolumn{4}{|c|}{ MS-provoked ischemia } \\
\hline & & & \multicolumn{2}{|c|}{ Yes $(N=64)$} & \multicolumn{2}{|c|}{ No $(N=97)$} \\
\hline & $\mathrm{BL}$ & MS & $\mathrm{BL}$ & MS & $\mathrm{BL}$ & MS \\
\hline SBP & ${ }^{*} 133.6 \pm 18.4$ & $159.5 \pm 21.0$ & ${ }^{*} 136.1 \pm 21.0$ & $160.1 \pm 23.6$ & ${ }^{*} 130.6 \pm 16.4$ & $159.1 \pm 19.6$ \\
\hline DBP & ${ }^{*} 75.2 \pm 10.1$ & $88.4 \pm 10.8$ & ${ }^{*} 76.8 \pm 10.7$ & $87.5 \pm 11.3$ & ${ }^{*} 74.3 \pm 9.7$ & $88.9 \pm 10.5$ \\
\hline HR & ${ }^{*} 59.1 \pm 11.0$ & $68.6 \pm 11.9$ & ${ }^{*} 61.3 \pm 13.3$ & $70.6 \pm 13.6$ & *57.8 \pm 9.3 & $67.4 \pm 10.7$ \\
\hline Epinephrine (pg/mL) & $28.7 \pm 38.3$ & $46.7 \pm 64.7$ & $35.0 \pm 50.6$ & $61.1 \pm 88.7$ & $24.6 \pm 26.8$ & $37.3 \pm 40.4$ \\
\hline Norepinephrine $(\mathrm{pg} / \mathrm{mL})$ & $365.5 \pm 259.2$ & $389.9 \pm 236.7$ & $402.6 \pm 263.2$ & $437.4 \pm 275.0$ & $341.0 \pm 254.9$ & $358.2 \pm 202.7$ \\
\hline
\end{tabular}

Values are mean \pm standard deviation. Changes from BL to MS in both SBP and DBP and HR were all significant at the ${ }^{*} p<0.0001$ level, however, the differences in BL or MS values between those with (YES) versus without (NO) MS-provoked ischemia were not statistically significant. There were no significant differences in epinephrine and norepinephrine across conditions or between groups. 
Furthermore, the graded incremental contribution of BMI highlights that increasing adiposity may contribute to vulnerability for transient, asymptomatic, yet prognostically relevant myocardial ischemia during mentally and emotionally provocative events during daily life. Thus, increasing BMI may serve as a vulnerability marker for this ischemic phenomenon.

There may be various reasons to account for the observed contribution of BMI to vulnerability for myocardial ischemia provoked by MS. Our group and others have shown that those patients vulnerable to myocardial ischemia induced by MS challenge have higher circulating C-reactive protein (CRP) and ET-1 and reduced parasympathetic tone during emotional provocation $(19,22)$. These are the constituents of the parasympathetic inflammatory reflex. We extended these observations to patients with a spectrum of BMI's. We reasoned that those with higher BMI have higher circulating inflammatory factors and autonomic dysregulation (components of metabolic syndrome) that provide a physiologic milieu that would increase risk to MS-induced myocardial ischemia.

For example, abdominal adiposity and BMI have been positively associated with certain psychological factors such as hostility, anxiety and depression (14-16), each of which is a risk marker for ACS, progression of CAD and stress-provoked ischemia $(17,23)$. Furthermore, several of the physiological consequences associated with adiposity, such as a higher chronic inflammatory state (12), autonomic dysregulation (24) and endothelial dysfunction (25), overlap with the conceptual mechanisms underlying this ischemic syndrome. For example, psychological stress acutely affects the activity of the autonomic nervous system in a manner that can facilitate inflammation (10), endothelial dysfunction and coronary microvascular dysfunction $(9,26,27)$. These effects of psychological stress might be exacerbated in obese individuals. Specifically, adiposity confers a heightened sympathetic tone
$(20,28,29)$, which could contribute to enhanced risk of coronary vasoconstriction because of synergy between norepinephrine and endothelin-1 (30), the most potent endogenous vasoconstrictor that is found in coronary plaque-resident macrophages (22). Similarly, we have previously reported that CRP, a marker of a chronic BL inflammatory state, increases risk for MS-provoked defect in myocardial perfusion (19), and higher levels of this marker are observed with increasing abdominal fat and general adiposity $(31,32)$. Furthermore, much of the sympathetically mediated cytokine production originates from adipose tissue (33). Thus, the higher BL sympathetic tone and potentiation of cytokine production associated with adiposity may contribute to a chronic state whereby the additional effects of MS on these pathways easily crosses the threshold necessary for the precipitation of a prognostically relevant deficit in myocardial perfusion.

The nature of the sample, which was predominantly male and Caucasian, limits the applicability of these findings, though the distribution of women and ethnic minorities was comparable for those with and without MS-provoked myocardial ischemia. In addition, the sample size did not allow us to test whether BMI accounted at least in part for our previous finding that chronic level of circulating CRP increased likelihood of MS-provoked myocardial ischemia in a dose-response manner (12). Furthermore, we did not have information on the fitness level of study participants, information that might have provided additional insights regarding BMI and risk of MS-provoked ischemia. Our findings are exploratory and should be corroborated by studies with larger and more diverse samples, using different anthropometric indices of fat deposition such as waist circumference, waistto-hip ratio and/or skinfold thickness.

\section{CONCLUSION}

We found that in a sample of patients with chronic, stable CAD, BMI was an independent vulnerability marker for a new or worsening myocardial perfusion deficit provoked by MS-MS-provoked myocardial ischemia. The pathophysiology underlying this finding is consistent with the autonomic and proinflammatory consequences of adiposity. Further research focusing on mechanistic underpinnings of these observations and in diverse populations may advance conceptual constructs regarding the complex effects of excess adiposity on cardiovascular health.

\section{ACKNOWLEDGMENTS}

This work was supported by R01 awards (HL59619-01 and HL071116-01) from the National Heart, Lung, and Blood Institute and by a Merit Review award from the Department of Veterans Affairs to Dr. R Soufer. The authors would like to acknowledge Dr. Hitender Jain for his contributions in organizing the $\mathrm{CV}$ Nuclear Medicine Studies for evaluation.

\section{DISCLOSURE}

The authors declare that they have no competing interests as defined by Molecular Medicine, or other interests that might be perceived to influence the results and discussion reported in this paper.

\section{REFERENCES}

1. Deanfield JE, et al. (1984) Silent myocardial ischaemia due to mental stress. Lancet. 2:1001-5.

2. Rozanski A, et al. (1998) Mental stress and the induction of silent myocardial ischemia in patients with coronary artery disease. N. Engl. J. Med. 318:1005-12.

3. Goldberg AD, et al. (1996) Ischemic, hemodynamic, and neurohormonal responses to mental and exercise stress. Experience from the Psychophysiological Investigations of Myocardial Ischemia Study (PIMI). Circulation. 94:2402-9.

4. Jiang W, et al. (1996) Mental stress-induced myocardial ischemia and cardiac events. JAMA. 275:1651-6.

5. Krantz DS, et al. (1999) Prognostic value of mental stress testing in coronary artery disease. Am. J. Cardiol. 84:1292-7.

6. Jain D, et al. (1995) Prognostic implications of mental stress-induced silent left ventricular dysfunction in patients with stable angina pectoris. Am. J. Cardiol. 76:31-35.

7. Sheps DS, et al. (2002) Mental stress-induced ischemia and all-cause mortality in patients 
with coronary artery disease: Results from the Psychophysiological Investigations of Myocardial Ischemia Study. Circulation. 105:1780-4.

8. Yeung AC, et al. (1991) The effect of atherosclerosis on the vasomotor response of coronary arteries to mental stress. N. Engl. J. Med. 325:1551-6.

9. Arrighi JA, et al. (2000) Myocardial blood-flow response during mental stress in patients with coronary artery disease. Lancet. 356:310-11.

10. Tracey KJ. (2002) The inflammatory reflex. Nature. 420:853-9.

11. Lewis CE, et al. (2009) Mortality, health outcomes, and body mass index in the overweight range: A science advisory from the American Heart Association. Circulation. 119:3263-71.

12. Tilg H, Moschen AR. (2006) Adipocytokines: Mediators linking adipose tissue, inflammation and immunity. Nat. Rev. Immunol. 6:772-83.

13. Patel AR, et al. (2009) Modestly overweight women have vascular endothelial dysfunction. Clin. Cardiol. 2009. 32:269-73.

14. Niaura R, et al. (2000) Hostility and the metabolic syndrome in older males: The normative aging study. Psychosom. Med. 62:7-16.

15. Almeida OP, Calver J, Jamrozik K, Hankey GJ, Flicker L. (2009) Obesity and metabolic syndrome increase the risk of incident depression in older men: The health in men study. Am. J. Geriatr. Psychiatry. 17:889-98.

16. Gariepy G, Nitka D, Schmitz N. (2009) The association between obesity and anxiety disorders in the population: A systematic review and meta-analysis. Int. J. Obes. 34:407-19.

17. Burg, MM, Jain D, Soufer R, Kerns RD, Zaret BL. (1993) Role of behavioral and psychological factors in mental stress-induced silent left ventricular dysfunction in coronary artery disease. J. Am. Coll. Cardiol. 22:440-8.

18. Rozanski A, Blumenthal JA, Kaplan J. (1999) Impact of psychological factors on the pathogenesis of cardiovascular disease and implications for therapy. Circulation. 99:2192-217.

19. Shah R, et al. (2006) C-reactive protein and vulnerability to mental stress-induced myocardial ischemia. Mol. Med. 12:269-74.

20. Brydon L, et al. (2008) Circulating leptin and stress-induced cardiovascular activity in humans. Obesity. 16:2642-7.

21. Holly TA, et al. (2010) Single photon-emission computed tomography. J. Nucl. Cardiol. 17:941-73.

22. Fernandez AB, et al. (2010) Tendency to angry rumination predicts stress-provoked endothelin-1 increase in patients with coronary artery disease. Psychosom. Med. 72:348-53.

23. Williams RB Jr, Suarez EC, Kuhn CM, Zimmerman EA, Schanberg SM. (1991) Biobehavioral basis of coronary-prone behavior in middle-aged men. Part I: Evidence for chronic SNS activation in Type As. Psychosom. Med. 53:517-27.

24. Rabbia, F, et al. (2003) Assessment of cardiac autonomic modulation during adolescent obesity. Obes. Res. 11:541-8.
25. Higashi $Y$, et al. (2001) Effect of obesity on endothelium-dependent, nitric oxide-mediated vasodilation in normotensive individuals and patients with essential hypertension. Am. J. Hypertens. 14:1038-45.

26. Spieker LE, et al. (2002) Mental stress induces prolonged endothelial dysfunction via endothelin-A receptors. Circulation. 105:2817-20.

27. Treiber FA, et al. (2000) Racial differences in endothelin- 1 at rest and in response to acute stress in adolescent males. Hypertension. 35:722-5.

28. Grassi, G, et al. (1995) Sympathetic activation in obese normotensive subjects. Hypertension. 25(4 Pt 1):560-3.

29. van Baak MA. (2001) The peripheral sympathetic nervous system in human obesity. Obes. Rev. 2:3-14.

30. Yang ZH, et al. (1990) Threshold concentrations of endothelin-1 potentiate contractions to norepinephrine and serotonin in human arteries. A new mechanism of vasospasm? Circulation. 82:188-95.

31. Tadros TM, et al. (2009) Pericardial fat volume correlates with inflammatory markers: The Framingham Heart Study. Obesity. 18:1039-45.

32. Aronson D, et al. (2004) Obesity is the major determinant of elevated C-reactive protein in subjects with the metabolic syndrome. Int. J. Obes. Relat. Metab. Disord. 28:674-9.

33. Mohamed-Ali V, et al. (2001) Beta-adrenergic regulation of IL-6 release from adipose tissue: In vivo and in vitro studies. J. Clin. Endocrinol. Metab. 86:5864-9.

Cite this article as: Soufer $R$, Fernandez $A B$, Meadows J, Collins D, Burg MM. (2016) Body mass index and risk for mental stress-induced ischemia in coronary artery disease. Mol. Med. 22:286-91. 